Archive for

Organic Chemistry

Arkivoc 2020, part vi, 114-125

\title{
One-pot, regioselective synthesis of functionalized indole derivatives: a three- component domino reaction of arylamine, arylglyoxal, and 4-hydroxycoumarin or 4-hydroxy-6-methyl-2-pyrone
}

\author{
Hossein Mehrabi*, Fatemeh Dastouri, Sara Asadi, Farzaneh Alizadeh-Bami, and Reza Ranjbar-Karimi \\ Department of Chemistry, Vali-e-Asr University of Rafsanjan, 77176 Rafsanjan, Iran \\ E-mail: mehraby h@yahoo.com
}

Received 01-11-2020

Accepted 04-21-2020

Published on line 05-01-2020

\section{Abstract}

A metal free, one-pot tandem synthetic routes for functionalized indole derivatives has been established. An efficient three-component reaction was designed with incorporation of Knoevenagel condensation followed by inter-, and intramolecular nucleophilic addition reaction in one-pot under mild condition. The structural diversities of the synthesized compounds have been confirmed spectroscopically, by IR, ${ }^{1} \mathrm{H}$ - and ${ }^{13} \mathrm{C} N M R$, and elemental analyses which agree with the proposed structures.

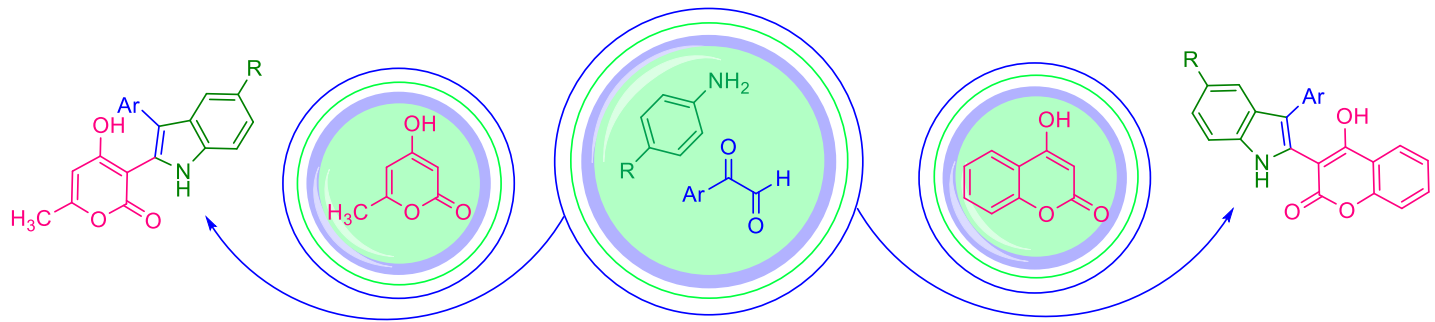

Keywords: Arylamine, Arylglyoxal, 4-Hydroxycoumarin, 4-Hydroxy-6-methyl-2-pyrone, Functionalized indole derivatives 


\section{Introduction}

The indole motifs are one of the most important and abundant nitrogen-containing heterocyclic compounds found in important natural products, various functional molecules, and pharmaceuticals. ${ }^{1}$ Indole analogs are very interesting to organic and medicinal chemists due to their biological activities, such as, anti-inflammatory $\mathbf{A},{ }^{2}$ antimicrobial $\mathbf{B},{ }^{3}$ antioxidant $\mathbf{C},{ }^{4}$ and antibacterial $\mathbf{D}$ activity (Figure 1$){ }^{5}$

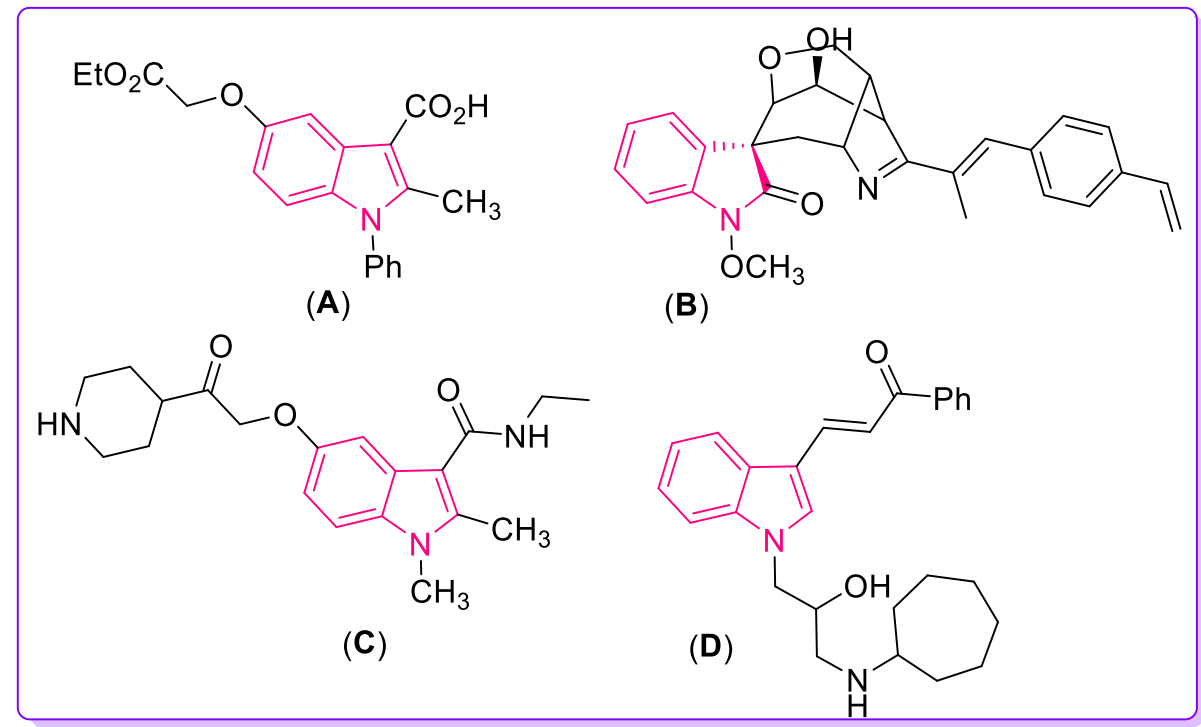

Figure 1. Selected bioactive molecules indoles.

In addition, many indole derivatives have exhibited high cytotoxic activity against breast, esophageal, colonic, lung, and stomach tumor cell lines. ${ }^{6,7}$ Moreover, indole cores can be found in the structure of different drugs such as sumatriptan, ${ }^{8}$ reserpine, ${ }^{9}$ and lotronex. ${ }^{10}$ On the other hand, among indole and its structural analogs, especially indolyl chromene and indolyl pyran derivatives, are found in a large number of pharmacological activities, such as antibacterial $\mathbf{E}{ }^{11}$ antimicrobial $\mathbf{F},{ }^{12,13}$ anticancer $\mathbf{G},{ }^{14}$ antioxidant $\mathbf{H},{ }^{15,16}$ and antifungal I activities (Figure 2). ${ }^{17}$ 


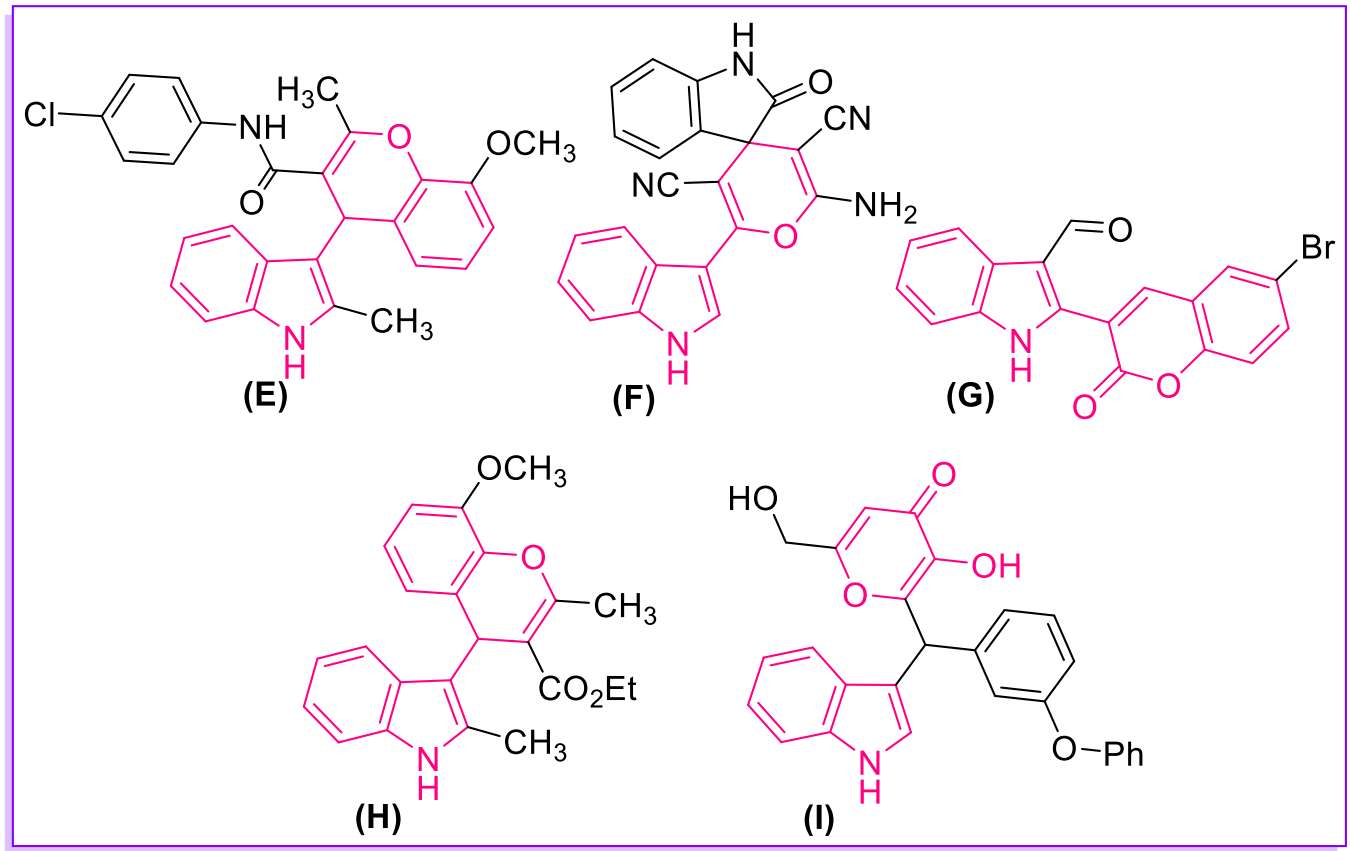

Figure 2. Selected examples of bioactive indolyl chromene and indolyl pyran derivatives.

Therefore, studies on strategies for the synthesis of indole derivatives have received high importance in organic synthesis. In this regard, there are many traditional methods for the preparation of indole derivatives including Fischer reaction, ${ }^{18,19}$ Bischler reaction, ${ }^{20}$ Reissert reaction, ${ }^{21}$ Bartoli reaction, ${ }^{22}$ and LeimgruberBatcho reaction. ${ }^{23}$ Recently, new strategies have been developed for the synthesis of indole derivatives; these include: various metal-catalyzed, ${ }^{24-29}$ multi-component method, ${ }^{30}$ cyclization reaction, ${ }^{31}$ Wittig reactions, ${ }^{32}$ and various other methods. ${ }^{33-36}$ Most of these protocols appear to suffer from some drawbacks such as tedious experimental procedures, difficult reaction conditions, multiple synthetic steps, low yields, and usage of expensive catalysts and reagents. Therefore, the development of more economic, convenient and efficient approaches to the regioselective synthesis of substituted indoles under mild conditions is still an attractive proposition. In continuation of our previous works on the application of arylglyoxal in synthesis of heterocyclic compounds, ${ }^{37,38}$ we herein report new synthetic strategy for the domino arylamine, arylglyoxal, and 4hydroxycoumarin or 4-hydroxy-6-methyl-2-pyrone yielding the synthesis of functionalized indole derivatives with high yields.

\section{Results and Discussion}

To find the optimized conditions, we studied the synthesis of 4-hydroxy-3-(5-methoxy-3-(p-tolyl)-1H-indol-2$\mathrm{yl}$ )-2H-chromen-2-one $4 \mathrm{a}$ via the three-component reaction of 4-methoxyaniline 1a, 4-methylphenylglyoxal $\mathbf{2 a}$, and 4-hydroxycoumarin $\mathbf{3}$ under a variety of conditions (Table 1). 
Table 1. Optimization of the reaction conditions

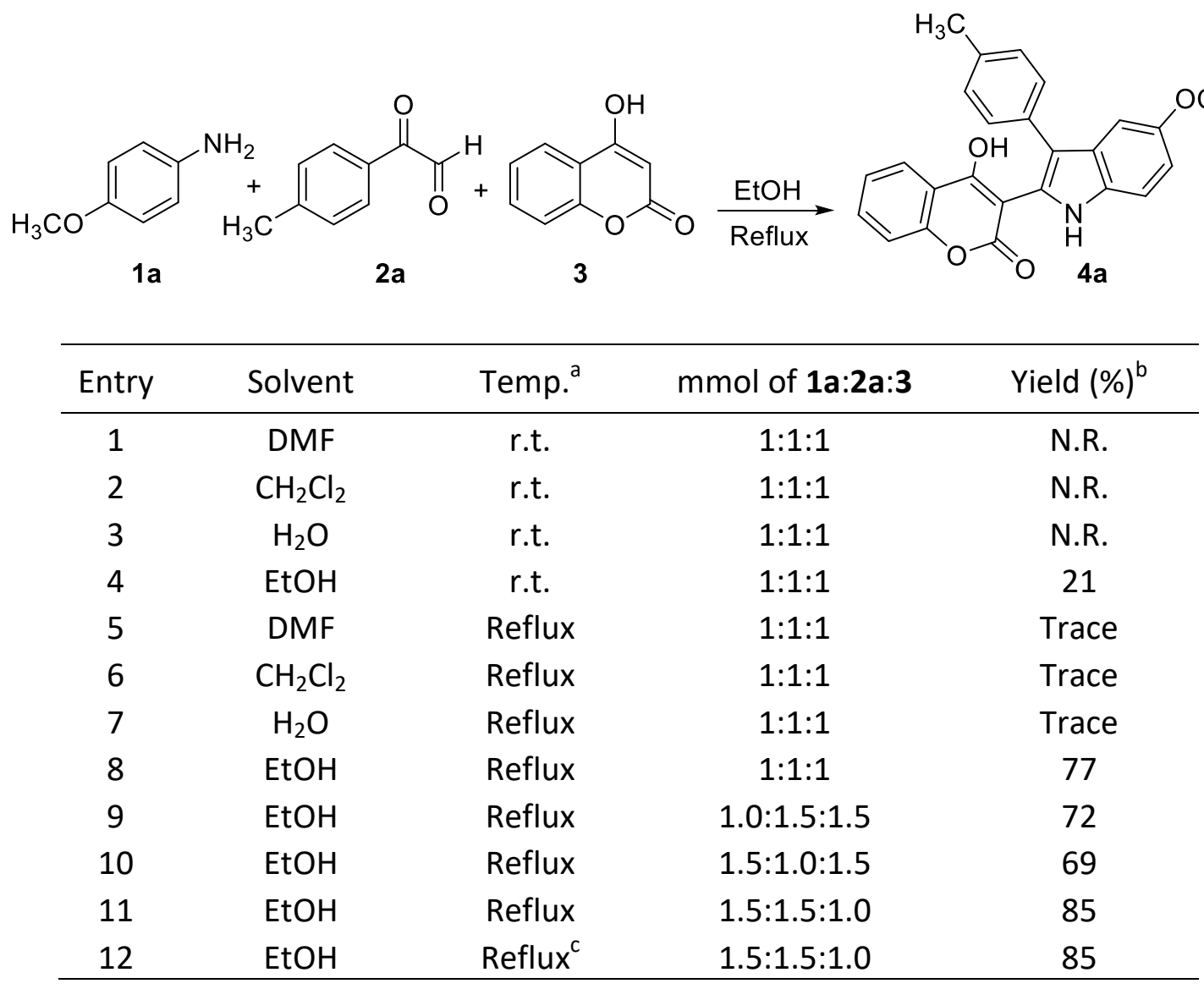

${ }^{\text {a }}$ Reaction conditions: solvent was $15 \mathrm{~mL}$, reaction time was $10 \mathrm{~h}$.

${ }^{\mathrm{b}}$ Isolated yields.

${ }^{\mathrm{c}}$ Reaction time was $24 \mathrm{~h}$.

The optimization of the reaction conditions, including the solvent, temperature, and the equivalents of starting materials were investigated. First, various solvents were examined (Table 1, entries 1-4), and ethanol was found to be the preeminent solvent for this reaction. Then, we examined the influence of different temperatures on this reaction. When the reaction was carried out for $10 \mathrm{~h}$. at room temperature, the product formed in $21 \%$ yield, but under reflux conditions at the same time, the product was formed in $77 \%$ yield (Table 1 , entries 4 and 8). Finally, we observed that the amount of starting materials also have important influence on the reaction (Table 1, entries 8-11). A larger amount of 4-methoxyaniline 1a, and 4-methylphenylglyoxal 2a (for example, $1.5 \mathrm{mmol}$ ) in ethanol at reflux temperature resulted in a higher yield, 85\% (Table 1, entry 11). Also, increasing the reaction time in ethanol under reflux condition did not improve the yield (Table 1, entry 12). This series of experiments reveal that the optimal results were obtained when the reaction of 4methylphenylglyoxal 2a (1.5 mmol) was conducted with 4-methoxyaniline $1 \mathrm{a}$ (1.5 mmol), and 4hydroxycoumarin $3(1.0 \mathrm{mmol})$ in ethanol under reflux conditions. These optimized reaction conditions (Table 1 , entry 11) were then used to synthesize and explore the scope of this novel transformation with various arylamine, arylglyoxal, and 1,3-dicarbonyl compounds such as 4-hydroxycoumarin and 4-hydroxy-6-methyl-2pyrone, to give two series of 4-hydroxy-3-(3-aryl-1H-indol-2-yl)-2H-pyran-2-ones 4a-i and 4-hydroxy-3-(3-aryl$1 \mathrm{H}$-indol-2-yl)-2H-chromen-2-ones 6a-f in good yields (Table 2). 
As can be seen from Table 2, the nature of the arylamine, the arylglyoxal, and the 1,3-dicarbonyl compounds was important. When the arylamine derivatives especially with electron-donating groups, the arylglyoxal derivatives with electron-withdrawing groups, and the 1,3-dicarbonyl compounds such as 4hydroxycoumarin were employed, a higher yield was achieved.

Table 2. Synthesis of 4-hydroxy-3-(3-aryl-1H-indol-2-yl)-2H-pyran-2-ones and 4-hydroxy-3-(3-aryl-1H-indol-2yl)-2H-chromen-2-ones

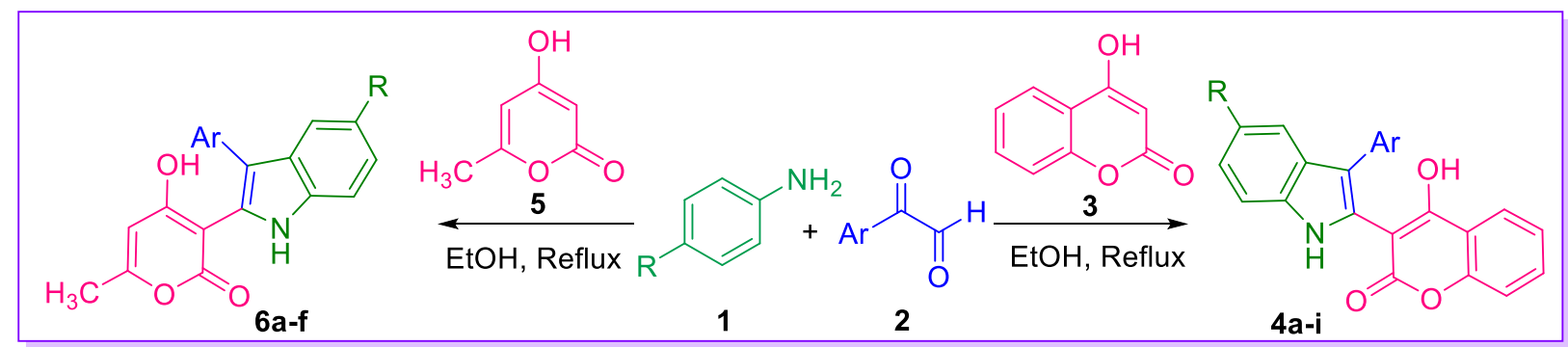

\begin{tabular}{|c|c|c|c|c|c|}
\hline Entry & Product $^{a}$ & $\mathrm{R}$ & $\mathrm{Ar}$ & 3 or 5 & Yield $(\%)^{b}$ \\
\hline 1 & $4 a$ & $\mathrm{OCH}_{3}$ & $4-\mathrm{CH}_{3} \mathrm{C}_{6} \mathrm{H}_{4}$ & & 85 \\
\hline 2 & $4 b$ & $\mathrm{OCH}_{3}$ & $4-\mathrm{ClC}_{6} \mathrm{H}_{4}$ & & 93 \\
\hline 3 & 4c & $\mathrm{OCH}_{3}$ & 4- $\mathrm{BrC}_{6} \mathrm{H}_{4}$ & & 91 \\
\hline 4 & $4 d$ & $\mathrm{OCH}_{3}$ & $4-\mathrm{FC}_{6} \mathrm{H}_{4}$ & & 94 \\
\hline 5 & $4 e$ & $\mathrm{Cl}$ & $4-\mathrm{OCH}_{3} \mathrm{C}_{6} \mathrm{H}_{4}$ & & 79 \\
\hline 6 & $4 f$ & $\mathrm{Br}$ & $4-\mathrm{OCH}_{3} \mathrm{C}_{6} \mathrm{H}_{4}$ & & 78 \\
\hline 7 & $4 g$ & $\mathrm{Br}$ & $4-\mathrm{NO}_{2} \mathrm{C}_{6} \mathrm{H}_{4}$ & & 80 \\
\hline 8 & $4 h$ & $\mathrm{CH}_{3}$ & $3,4-\left(\mathrm{OCH}_{3}\right)_{2} \mathrm{C}_{6} \mathrm{H}_{3}$ & & 82 \\
\hline 9 & $4 \mathbf{i}$ & $\mathrm{Cl}$ & $2-\mathrm{CH}_{3} \mathrm{C}_{6} \mathrm{H}_{4}$ & & 78 \\
\hline 10 & $6 a$ & $\mathrm{CH} 3$ & 4- $\mathrm{CH} 3 \mathrm{C} 6 \mathrm{H} 4$ & & 59 \\
\hline 11 & $6 b$ & $\mathrm{CH} 3$ & $4-\mathrm{ClC} 6 \mathrm{H} 4$ & & 62 \\
\hline 12 & $6 c$ & $\mathrm{OCH} 3$ & 4-ClC6H4 & & 68 \\
\hline
\end{tabular}


Table 2. Continued

\begin{tabular}{ccccc}
\hline Entry & Product $^{\mathrm{a}}$ & $\mathrm{R}$ & $\mathrm{Ar}$ & ${\text { Yield }(\%)^{\mathrm{b}}}_{13}$ \\
$\mathbf{6 d}$ & $\mathrm{CH}_{3}$ & $4-\mathrm{NO}_{2} \mathrm{C}_{6} \mathrm{H}_{4}$ & \\
14 & $6 \mathrm{e}$ & $\mathrm{CH}_{3}$ & $4-\mathrm{OCH}_{3} \mathrm{C}_{6} \mathrm{H}_{4}$ & \\
15 & $6 f$ & $\mathrm{Cl}$ & $2-\mathrm{CH}_{3} \mathrm{C}_{6} \mathrm{H}_{4}$ & or 5 \\
\hline
\end{tabular}

${ }^{a}$ Reaction conditions: 1 (1.5 mmol), 2 (1.5 mmol), 3 or $5(1.0 \mathrm{mmol})$; solvent volume $15.0 \mathrm{~mL}$ and reaction time was $10 \mathrm{~h}$.

${ }^{\mathrm{b}}$ Isolated yields.

All the synthesized compounds were unknown, and were characterized by ${ }^{1} \mathrm{H}$ and ${ }^{13} \mathrm{C} N M R, I R, C H N$ analysis and melting points. For instance, the ${ }^{1} \mathrm{H}$ NMR spectrum of the compound 4 a consisted of one singlet at $\delta=2.65 \mathrm{ppm}$ for the three hydrogens of the methyl group. A singlet that integrated for three protons was observed at $\delta=3.33 \mathrm{ppm}$ for the methoxy protons. The aromatic protons resonated in the region $\delta=6.29-$ $7.49 \mathrm{ppm}$, and a broad singlet that integrated for one hydrogen was observed at $\delta=9.71 \mathrm{ppm}$ for the hydroxyl proton. A broad singlet at $\delta=10.52 \mathrm{ppm}$ for the proton of the nitrogen group. The ${ }^{13} \mathrm{C}$ NMR spectrum of compound $4 \mathrm{a}$ exhibited 23 distinct signals in agreement with the proposed structure. Partial assignments of these resonances for the other products are given in the experimental section.

Based on our experimental results and literature reports, we proposed a possible mechanism for the formation of 4-hydroxy-3-(3-aryl-1H-indol-2-yl)-2H-pyran-2-ones 4 and 4-hydroxy-3-(3-aryl-1H-indol-2-yl)-2Hchromen-2-ones 6 as shown in Scheme 1. Firstly, intermediate $\mathbf{J}$ is formed through Knoevenagel condensation of the arylamine $\mathbf{1}$ to the arylglyoxal $\mathbf{2}$. Then, this intermediate is converted into intermediate $\mathbf{K}$ through a intermolecular nucleophilic addition reaction, which subsequently undergoes an intramolecular nucleophilic addition reaction to form intermediate $L$. In the last step, intermediate $\mathbf{L}$ afforded the desired products $\mathbf{4}$ and $\mathbf{6}$ in good yield with removal of $\mathrm{H}_{2} \mathrm{O}$ and $[1,3-\mathrm{H}]$ shift. 


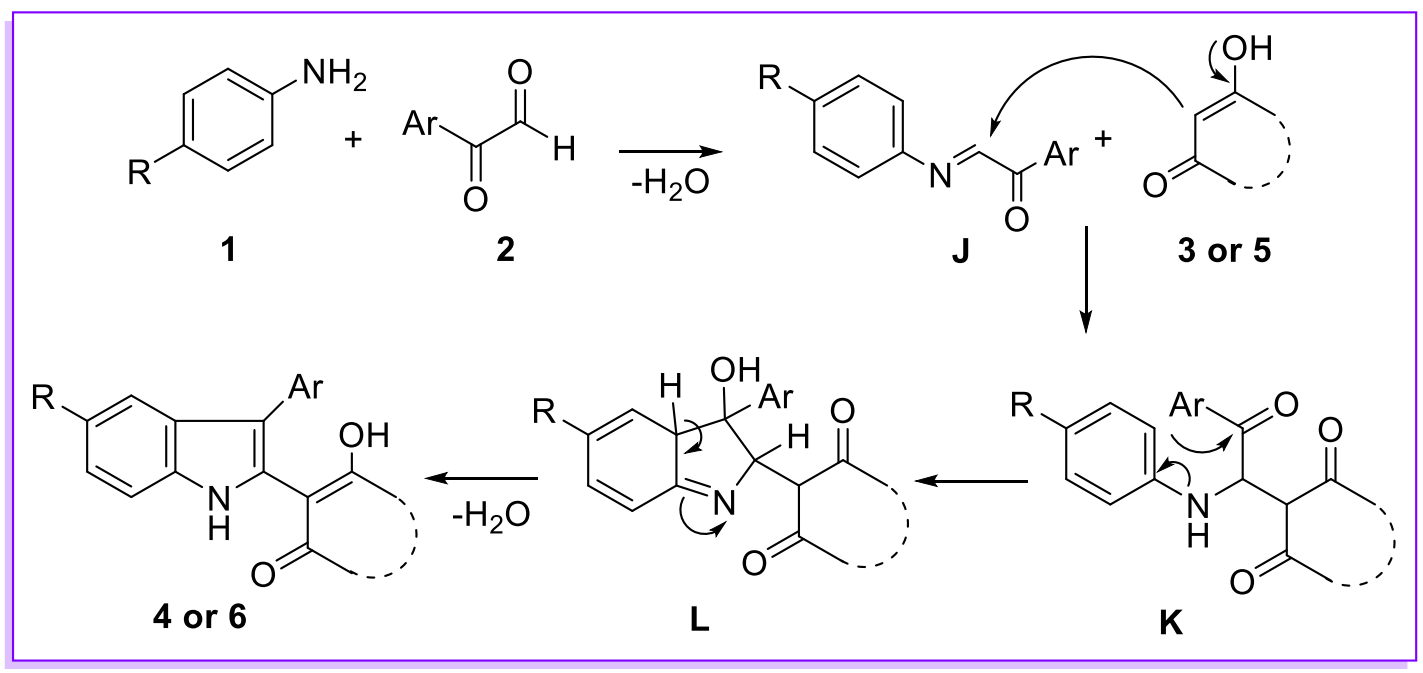

Scheme 1. The proposed mechanism for formation of 4-hydroxy-3-(3-aryl-1H-indol-2-yl)-2H-pyran-2-ones and 4-hydroxy-3-(3-aryl-1H-indol-2-yl)-2H-chromen-2-ones.

\section{Conclusions}

In summary, we have reported the development of a facile synthesis of functionalized indol derivatives using a one-pot, three-component process through Knoevenagel condensation followed by inter-, and intramolecular nucleophilic addition reaction. Using this method, we were able to assemble a wide range of indol derivatives with good to excellent yields in a single step. The salient feature of our protocol is high atom-economy, operational simplicity, easy work-up, and easily available precursors.

\section{Experimental Section}

General. All chemicals were purchased from Aldrich and Merck with high-grade quality, and used without any purification. All melting points were obtained by Barnstead Electrothermal 9200 apparatus and are uncorrected. The reactions were monitored by TLC and all yields refer to isolated products. NMR spectra were obtained on a Varian $500 \mathrm{MHz}$ spectrometer $\left({ }^{1} \mathrm{H} \mathrm{NMR}\right.$ at $500 \mathrm{MHz},{ }^{13} \mathrm{C} N M R$ at $\left.125 \mathrm{MHz}\right)$ in DMSO using TMS as an internal standard. Infrared spectra were recorded on a Bruker FT-IR Equinax-55 spectrophotometer in $\mathrm{KBr}$ with absorption in $\mathrm{cm}^{-1}$. Elemental analyses were performed using a Carlo Erba EA 1108 instrument. All products were characterized by their spectral and physical data.

General procedure for the synthesis of compounds 4a-i. A mixture of arylamine 1 (1.5 mmol) and arylglyoxal 2 $(1.5 \mathrm{mmol}$ ) was stirred in $15 \mathrm{~mL}$ of ethanol at reflux for $2 \mathrm{~h}$. to give iminone. Then, 4-hydroxycoumarin 3 (1.0 $\mathrm{mmol}$ ) was added, and obtained mixture was refluxed for $8 \mathrm{~h}$. After completion of the reaction, determined by TLC, the solvent was removed under reduced pressure, and the resulting crude product was purifed by washing with diethyl ether then, by simple filtration and washing with cold ethyl acetate to give the pure compounds 4a-i (78-94\%).

General procedure for the synthesis of compounds 6a-f. 
A mixture of arylamine $1(1.5 \mathrm{mmol})$ and arylglyoxal $2(1.5 \mathrm{mmol})$ was stirred in $15 \mathrm{~mL}$ of ethanol at reflux for 2 h. to give iminone. Then, 4-hydroxy-6-methyl-2-pyrone $5(1.0 \mathrm{mmol})$ was added, and obtained mixture was refluxed for $8 \mathrm{~h}$. After completion of the reaction, determined by TLC, the solvent was removed under reduced pressure, and the viscous residue was purified by plate chromatography $(20 \times 20 \mathrm{~cm})$ using n-hexane/EtOAc $(2: 1)$ as eluent to give the pure compounds $6 a-f(55-68 \%)$.

3-(5-Methoxy-3-(p-tolyl)-1H-indol-2-yl)chromane-2,4-dione (4a). White powder; mp 260-263 ${ }^{\circ} \mathrm{C} . \mathrm{IR} \mathrm{v}^{\mathrm{v}} / \mathrm{cm}^{-1}$ $(\mathrm{KBr}): 3026,1763,1601,1444 \mathrm{~cm}^{-1} .{ }^{1} \mathrm{H} N M R(500 \mathrm{MHz}, \mathrm{DMSO}): \delta 2.65\left(\mathrm{~s}, 3 \mathrm{H}, \mathrm{CH}_{3}\right), 3.33\left(\mathrm{~s}, 3 \mathrm{H}, \mathrm{OCH}_{3}\right), 6.29(\mathrm{~s}$, $1 \mathrm{H}, \mathrm{ArH}), 6.36(\mathrm{~d}, J 11.0 \mathrm{~Hz}, 1 \mathrm{H}, \mathrm{ArH}), 6.69(\mathrm{~d}, J 8.0 \mathrm{~Hz}, 2 \mathrm{H}, \mathrm{ArH}), 6.88(\mathrm{t}, J 7.5 \mathrm{~Hz}, 1 \mathrm{H}, \mathrm{ArH}), 6.92(\mathrm{~d}, J 8.5 \mathrm{~Hz}, 2 \mathrm{H}$, $\operatorname{ArH}), 7.08(\mathrm{~d}, J 8.0 \mathrm{~Hz}, 2 \mathrm{H}, \mathrm{ArH}), 7.16(\mathrm{t}, J 7.5 \mathrm{~Hz}, 1 \mathrm{H}, \mathrm{ArH}), 7.48(\mathrm{~d}, J 7.5 \mathrm{~Hz}, 1 \mathrm{H}, \mathrm{ArH}), 9.71(\mathrm{~s}, 1 \mathrm{H}, \mathrm{OH}), 10.52(\mathrm{~s}$, $1 \mathrm{H}, \mathrm{NH})$ ppm. ${ }^{13} \mathrm{C}$ NMR (125 MHz, DMSO): $\delta$ 21.1, 60.2, 102.7, 103.5, 116.6, 124.0, 126.8, 127.0, 129.5, 131.8, $132.6,134.7,135.1,137.1,137.8,144.6,145.1,146.9,150.2,154.4,159.1,159.2,171.0$ ppm. Anal. Calcd for $\mathrm{C}_{25} \mathrm{H}_{19} \mathrm{NO}_{4}$ (397.43): C, 75.55; H, 4.82; N, 3.52. Found: $\mathrm{C}, 75.73 ; \mathrm{H}, 4.86 ; \mathrm{N}, 3.49$.

3-(3-(4-Chlorophenyl)-5-methoxy-1H-indol-2-yl)chromane-2,4-dione (4b). White powder; $\mathrm{mp} 298-301{ }^{\circ} \mathrm{C}$. IR $\mathrm{v} / \mathrm{cm}^{-1}(\mathrm{KBr}): 3311,1684,1614,1485 \mathrm{~cm}^{-1} .{ }^{1} \mathrm{H}$ NMR (500 MHz, DMSO): $\delta 3.68\left(\mathrm{~s}, 3 \mathrm{H}, \mathrm{OCH}_{3}\right), 6.64(\mathrm{~s}, 1 \mathrm{H}, \mathrm{ArH})$, $6.74(\mathrm{~d}, J 6.5 \mathrm{~Hz}, 1 \mathrm{H}, \mathrm{ArH}), 7.25-7.31(\mathrm{~m}, 5 \mathrm{H}, \mathrm{ArH}), 7.52-7.56(\mathrm{~m}, 3 \mathrm{H}, \mathrm{ArH}), 7.87(\mathrm{t}, J 7.0 \mathrm{~Hz}, 1 \mathrm{H}, \operatorname{ArH}), 10.45(\mathrm{~s}$, $1 \mathrm{H}, \mathrm{OH}), 11.25$ (s, 1H, NH) ppm. ${ }^{13} \mathrm{C}$ NMR (125 MHz, DMSO): $\delta 55.8,99.5,101.1,101.1,103.0,112.6,112.7$, 116.6, 124.1, 124.4, 128.6, 129.0, 129.9, 132., 132.30, 132.4, 132.6, 136.5, 153.1, 154.1, 162.2, 162.5 ppm. Anal. Calcd for $\mathrm{C}_{24} \mathrm{H}_{16} \mathrm{CINO}_{4}$ (417.85): C, 68.99; H, 3.86; N, 3.35. Found: C, 69.10; H, 3.87; N, 3.39.

3-(3-(4-Bromophenyl)-5-methoxy-1H-indol-2-yl)chromane-2,4-dione (4c). White powder; mp $225-228{ }^{\circ} \mathrm{C}$. IR $\mathrm{v} / \mathrm{cm}^{-1}(\mathrm{KBr}): 3064,1679,1631,1482 \mathrm{~cm}^{-1} .{ }^{1} \mathrm{H}$ NMR $(500 \mathrm{MHz}, \mathrm{DMSO}): \delta 3.67\left(\mathrm{~s}, 3 \mathrm{H}, \mathrm{CH}_{3}\right), 6.83(\mathrm{~d}, J 8.0,2 \mathrm{H}$, $\mathrm{ArH}), 7.11(\mathrm{~d}, J 6.5 \mathrm{~Hz}, 2 \mathrm{H}, \mathrm{ArH}), 7.35$ (s, 2H, Ar-H), 7.67-7.81 (m, 6H, ArH, OH), $8.04(\mathrm{~s}, 1 \mathrm{H}, \mathrm{NH}) \mathrm{ppm} .{ }^{13} \mathrm{C} \mathrm{NMR}$ (125 MHz, DMSO): $\delta 55.8,96.4,110.4,114.8,117.4,120.2,121.5,124.9,127.3,127.4,128.4,129.1,130.0$, $130.4,130.5,132.7,134.6,135.7,159.7,176.7,181.6,184.8$ ppm. Anal. Calcd for $\mathrm{C}_{24} \mathrm{H}_{16} \mathrm{BrNO}_{4}$ (462.30): C, $62.35 ; \mathrm{H}, 3.49 ; \mathrm{N}, 3.03$. Found: $\mathrm{C}, 62.17 ; \mathrm{H}, 3.45 ; \mathrm{N}, 3.07$.

3-(3-(4-Fluorophenyl)-5-methoxy-1H-indol-2-yl)chromane-2,4-dione (4d). White powder; mp $162-165{ }^{\circ} \mathrm{C}$. IR $\mathrm{v} / \mathrm{cm}^{-1}(\mathrm{KBr}): 3041,1634,1595,1438 \mathrm{~cm}^{-1} .{ }^{1} \mathrm{H}$ NMR $(500 \mathrm{MHz}, \mathrm{DMSO}): \delta 3.67\left(\mathrm{~s}, 3 \mathrm{H}, \mathrm{CH}_{3}\right), 6.83(\mathrm{~m}, 12 \mathrm{H}, \mathrm{ArH}$, $\mathrm{OH}), 8.06$ (s, $1 \mathrm{H}, \mathrm{NH}) \mathrm{ppm} ;{ }^{13} \mathrm{C}$ NMR $(125 \mathrm{MHz}): \delta 55.6,114.6,115.7,116.7,116.8,117.4,120.2,124.9,126.1$, $127.4,127.5,127.5,128.5,128.5,131.6,131.6,135.6,154.3,159.3,164.7,168.7,168.7$ ppm. Anal. Calcd for $\mathrm{C}_{24} \mathrm{H}_{16} \mathrm{FNO}_{4}$ (401.39): C, 71.82; H, 4.02; N, 3.49. Found: $\mathrm{C}, 71.90 ; \mathrm{H}, 4.05 ; \mathrm{N}, 3.46$.

3-(5-Chloro-3-(4-methoxyphenyl)-1H-indol-2-yl)chromane-2,4-dione (4e). White powder; mp 271-274 ${ }^{\circ} \mathrm{C}$. IR $\mathrm{v} / \mathrm{cm}^{-1}$ (KBr): 3324, 1701, 1610, 1468, $\mathrm{cm}^{-1} .{ }^{1} \mathrm{H} \mathrm{NMR}$ (500 MHz, DMSO): $\delta 3.74\left(\mathrm{~s}, 3 \mathrm{H}, \mathrm{OCH}_{3}\right), 6.97(\mathrm{~d}, J 9 \mathrm{~Hz}, 2 \mathrm{H}$, $\mathrm{ArH}), 7.11(\mathrm{~d}, J 6.5 \mathrm{~Hz}, 1 \mathrm{H}, \mathrm{ArH}), 7.21(\mathrm{~s}, 1 \mathrm{H}, \mathrm{ArH}), 7.37(\mathrm{t}, J 7.5 \mathrm{~Hz}, 1 \mathrm{H}, \mathrm{ArH}), 7.43(\mathrm{~d}, J 8.5 \mathrm{~Hz}, 2 \mathrm{H}, \mathrm{ArH}), 7.54(\mathrm{~d}, J$ $8.5 \mathrm{~Hz}, 2 \mathrm{H}, \mathrm{ArH}) 7.66(\mathrm{t}, J 8.5 \mathrm{~Hz}, 1 \mathrm{H}, \mathrm{ArH}), 7.90(\mathrm{~d}, J 9.0 \mathrm{~Hz}, 1 \mathrm{H}, \mathrm{ArH}), 11.07(\mathrm{~s}, 1 \mathrm{H}, \mathrm{OH}), 11.71(\mathrm{~s}, 1 \mathrm{H}, \mathrm{NH}) \mathrm{ppm}$. ${ }^{13} \mathrm{C}$ NMR (125 MHz, DMSO): $\delta$ 55.5, 99.1, 101.5, 113.0, 114.6, 116.6, 118.3, 121.5, 124.1, 124.1, 124.3, 125.3, $128,5,131.0,132.5,135.1,139.0,153.2,159.4,162.2,162.6,170.7$ ppm.

3-(5-Bromo-3-(4-methoxyphenyl)-1H-indol-2-yl)chromane-2,4-dione (4f). Yellow powder; mp 291-294 ${ }^{\circ} \mathrm{C}$. IR $\mathrm{v} / \mathrm{cm}^{-1}(\mathrm{KBr}): 3319,1701,1610,1467,1411, \mathrm{~cm}^{-1} .{ }^{1} \mathrm{H}$ NMR $(500 \mathrm{MHz}, \mathrm{DMSO}): \delta 3.73\left(\mathrm{~s}, 3 \mathrm{H}, \mathrm{CH}_{3}\right), 6.96(\mathrm{~d}, J$ $8.5 \mathrm{~Hz}, 2 \mathrm{H}, \mathrm{ArH}), 7.21(\mathrm{~d}, J 6.5 \mathrm{~Hz}, 1 \mathrm{H}, \mathrm{ArH}) 7.34-7.43(\mathrm{~m}, 3 \mathrm{H}, \mathrm{ArH}), 7.42(\mathrm{~d}, J 8.5 \mathrm{~Hz}, 1 \mathrm{H}, \mathrm{ArH}), 7.52(\mathrm{~d}, J 9.0 \mathrm{~Hz}$, $2 \mathrm{H}, \mathrm{ArH}), 7.65(\mathrm{t}, J 8.5 \mathrm{~Hz}, 1 \mathrm{H}, \mathrm{ArH}) 7.89(\mathrm{~d}, J 6.5 \mathrm{~Hz}, 1 \mathrm{H}, \mathrm{ArH}) 11.05(\mathrm{~s}, 1 \mathrm{H}, \mathrm{OH}), 11.70(\mathrm{~s}, 1 \mathrm{H}, \mathrm{NH}) \mathrm{ppm} .{ }^{13} \mathrm{C} \mathrm{NMR}$ (125 MHz, DMSO): $\delta 55.5,112.0,113.4,114.6,116.6,116.7,121.3,123.9,124.0,124.1,124.2,125.2,128.5$, $128.6,131.7,132.5,135.3,138.8,153.2,159.4,162.2,170.7 \mathrm{ppm}$.

3-(5-Bromo-3-(4-nitrophenyl)-1H-indol-2-yl)chromane-2,4-dione (4g). White powder; mp $303-306{ }^{\circ} \mathrm{C} . \mathrm{IR}$ $\mathrm{v} / \mathrm{cm}^{-1}$ (KBr): 3087, 1658, 1600, $1493 \mathrm{~cm}^{-1} .{ }^{1} \mathrm{H}$ NMR (500 MHz, DMSO): $\delta 6.85$ (d, J 8.5Hz, 2H, ArH), 7.31-7.48 
$(\mathrm{m}, 5 \mathrm{H}, \mathrm{OH}, \mathrm{ArH}), 7.68(\mathrm{t}, J 8.5 \mathrm{~Hz}, 1 \mathrm{H}, \mathrm{ArH}), 7.85(\mathrm{~d}, J 9.0 \mathrm{~Hz}, 1 \mathrm{H}, \mathrm{ArH}), 7.93(\mathrm{~d}, J 7.5 \mathrm{~Hz}, 1 \mathrm{H}, \operatorname{ArH}), 8.27(\mathrm{~d}, J$ 9.0Hz, 2H, ArH), 12.15 (s, $1 \mathrm{H}, \mathrm{NH}$ ) ppm. ${ }^{13} \mathrm{C}$ NMR (125 MHz, DMSO): $\delta$ 98.5, 105.4, 112.7, 114.1, 116.7, 120.2, $122.3,124.2,124.3,124.5,125.8,127.9,131.1,132.3,132.8,136.0,139.2,146.7,153.3,162.0,162.9$ ppm. Anal. Calcd for $\mathrm{C}_{23} \mathrm{H}_{13} \mathrm{BrN}_{2} \mathrm{O}_{5}$ (477.27): $\mathrm{C}, 57.88 ; \mathrm{H}, 2.75 ; \mathrm{N}, 5.87$. Found: $\mathrm{C}, 57.79 ; \mathrm{H}, 2.74 ; \mathrm{N}, 5.90$.

3-(3(3,4-Dimethoxyphenyl)-5-methyl-1H-indol-2-yl)chromane-2,4-dione (4h). Yellow powder; mp 295-298 ${ }^{\circ} \mathrm{C}$. IR v $/ \mathrm{cm}^{-1}$ (KBr): 2939, 1692, 1613, $1494 \mathrm{~cm}^{-1} .{ }^{1} \mathrm{H}$ NMR (500 MHz, DMSO): $\delta 2.33\left(\mathrm{~s}, 3 \mathrm{H}, \mathrm{CH}_{3}\right), 3.64(\mathrm{~s}, 3 \mathrm{H}$, $\left.\mathrm{OCH}_{3}\right), 3.72\left(\mathrm{~s}, 3 \mathrm{H}, \mathrm{OCH}_{3}\right), 6.94-6.98(\mathrm{~m}, 3 \mathrm{H}, \mathrm{ArH}), 7.13(\mathrm{~d}, J 6.5 \mathrm{~Hz}, 1 \mathrm{H}, \mathrm{ArH}), 7.24(\mathrm{~s}, 1 \mathrm{H}, \mathrm{ArH}), 7.33(\mathrm{~d}, J 8.0 \mathrm{~Hz}$, $1 \mathrm{H}, \mathrm{ArH}), 7.38(\mathrm{t}, J 7.0 \mathrm{~Hz}, 1 \mathrm{H}, \mathrm{ArH}), 7.44(\mathrm{~d}, J 8.5 \mathrm{~Hz}, 1 \mathrm{H}, \mathrm{ArH}), 7.66(\mathrm{t}, J 6.5 \mathrm{~Hz}, 1 \mathrm{H}, \mathrm{ArH}), 7.91(\mathrm{~d}, J 6.5 \mathrm{~Hz}, 1 \mathrm{H}$, $\mathrm{ArH}), 11.00(\mathrm{~s}, 1 \mathrm{H}, \mathrm{OH}), 11.39(\mathrm{~s}, 1 \mathrm{H}, \mathrm{NH}) \mathrm{ppm} .{ }^{13} \mathrm{C} \mathrm{NMR}(125 \mathrm{MHz}, \mathrm{DMSO}): \delta 21.6,55.6,55.9,100.1,101.2$, $110.9,111.2,112.4,116.6,116.6,118.7,119.6,123.3,124.0,124.5,126.0,127.9,130.0,132.5,135.0,137.2$, 148.7, 148.9, 153.1, 162.3, 162.4 ppm. Anal. Calcd for $\mathrm{C}_{26} \mathrm{H}_{21} \mathrm{NO}_{5}$ (427.46): C, 73.06; H, 4.95; N, 3.28. Found: C, $72.98 ; \mathrm{H}, 4.93 ; \mathrm{N}, 3.31$.

3-(5-Chloro-3-(o-tolyl)-1H-indol-2-yl)chromane-2,4-dione (4i). Yellow powder; mp 234-236 ${ }^{\circ} \mathrm{C} . \mathrm{IR}_{\mathrm{V}} \mathrm{v} / \mathrm{cm}^{-1}$ (KBr): 3315, 1715, 1608, 1467, 1411, $\mathrm{cm}^{-1} .{ }^{1} \mathrm{H}$ NMR (500 MHz, DMSO): $\delta 2.27$ (s, 3H, CH $), 7.02-7.11(\mathrm{~m}, 4 \mathrm{H}$, $\mathrm{ArH}), 7.15(\mathrm{~d}, J 7.0 \mathrm{~Hz}, 1 \mathrm{H}, \mathrm{ArH}), 7.29(\mathrm{~s}, 1 \mathrm{H}, \mathrm{ArH}), 7.31$ (d, J 8.0Hz, 1H, ArH), 7.40 (t, J 7.0Hz, 1H, ArH), 7.49 (d, J $8.5 \mathrm{~Hz}, 1 \mathrm{H}, \mathrm{ArH}), 7.71(\mathrm{t}, J 7.0 \mathrm{~Hz}, 1 \mathrm{H}, \mathrm{ArH}), 7.85(\mathrm{~d}, J 6.5 \mathrm{~Hz}, 1 \mathrm{H}, \mathrm{ArH}), 11.03(\mathrm{~s}, 1 \mathrm{H}, \mathrm{OH}), 11.47(\mathrm{~s}, 1 \mathrm{H}, \mathrm{NH}) \mathrm{ppm}$. ${ }^{13} \mathrm{C}$ NMR (125 MHz, DMSO): $\delta$ 20.4, 102.5, 104.7, 110.3, 111.7, 114.4, 116.2, 116.8, 117.3, 119.7, 122.3, 124.2, $124.8,125.3,127.6,131.4,133.7,135.2,138.2,144.2,149.6,157.4,159.3,161.6$ ppm.

4-Hydroxy-6-methyl-3-(5-methyl-3-(p-tolyl)-1H-indol-2-yl)-2H-pyran-2-one (6a). Yellow powder; mp $188-201$ ${ }^{\circ} \mathrm{C} . \mathrm{IR} \mathrm{v} / \mathrm{cm}^{-1}(\mathrm{KBr}): 3411,3117,1650,1442 \mathrm{~cm}^{-1} .{ }^{1} \mathrm{H}$ NMR (500 MHz, DMSO): $\delta 2.04\left(\mathrm{~s}, 3 \mathrm{H}, \mathrm{CH}_{3}\right), 2.10(\mathrm{~s}, 3 \mathrm{H}$, $\left.\mathrm{CH}_{3}\right), 2.15\left(\mathrm{~s}, 3 \mathrm{H}, \mathrm{CH}_{3}\right), 5.74(\mathrm{~s}, 1 \mathrm{H}, \mathrm{ArH}), 6.70(\mathrm{~d}, J 9.9 \mathrm{~Hz}, 1 \mathrm{H}, \mathrm{ArH}), 6.85(\mathrm{~s}, 1 \mathrm{H}, \mathrm{ArH}), 6.90(\mathrm{~d}, J 7.8 \mathrm{~Hz}, 2 \mathrm{H}, \mathrm{ArH})$, $7.05(\mathrm{~d}, J 8.1 \mathrm{~Hz}, 1 \mathrm{H}, \mathrm{ArH}), 7.26(\mathrm{~d}, J 5.1 \mathrm{~Hz}, 2 \mathrm{H}, \mathrm{ArH}), 9.22(\mathrm{~s}, 1 \mathrm{H}, \mathrm{OH}), 10.11(\mathrm{~s}, 1 \mathrm{H}, \mathrm{NH}) \mathrm{ppm} .{ }^{13} \mathrm{C} \mathrm{NMR}(125$ $\mathrm{MHz}, \mathrm{DMSO}): \delta$ 19.7, 21.0, 21.3, 100.5, 108.8, 110.8, 119.0, 123.6, 124.9, 126.0, 126.7, 128.2, 128.9, 130.3, 134.6, 136.6, 136.7, 161.0, 164.9, 166.6 ppm. Anal. Calcd for $\mathrm{C}_{22} \mathrm{H}_{19} \mathrm{NO}_{3}$ (345.40): C, 76.50; $\mathrm{H}, 5.54 ; \mathrm{N}, 4.06$. Found: $\mathrm{C}, 76.37 ; \mathrm{H}, 5.51 ; \mathrm{N}, 4.04$.

3-(3-(4-Chlorophenyl)-5-methyl-1H-indol-2-yl)-4-hydroxy-6-methyl-2H-pyran-2-one (6b). Yellow powder; $\mathrm{mp}$ 254-257 ${ }^{\circ} \mathrm{C}$. IR v/cm $\mathrm{cm}^{-1}(\mathrm{KBr}): 3397,3085,1651,1445 \mathrm{~cm}^{-1} .{ }^{1} \mathrm{H}$ NMR (500 MHz, DMSO): $\delta 2.22\left(\mathrm{~s}, 3 \mathrm{H}, \mathrm{CH}_{3}\right), 2.32$ $\left(\mathrm{s}, 3 \mathrm{H}, \mathrm{CH}_{3}\right), 6.08(\mathrm{~s}, 1 \mathrm{H}, \mathrm{ArH}), 6.92(\mathrm{~s}, 1 \mathrm{H}, \mathrm{ArH}), 6.93(\mathrm{~d}, J 9.0 \mathrm{~Hz}, 1 \mathrm{H}, \mathrm{ArH}), 7.27(\mathrm{~d}, J 8.5 \mathrm{~Hz}, 1 \mathrm{H}, \mathrm{ArH}), 7.45(\mathrm{~d}, J$ $8.5 \mathrm{~Hz}, 2 \mathrm{H}, \mathrm{ArH}), 7.56(\mathrm{~d}, J 8.5 \mathrm{~Hz}, 2 \mathrm{H}, \mathrm{ArH}), 11.07(\mathrm{~s}, 1 \mathrm{H}, \mathrm{OH}), 11.34(\mathrm{~s}, 1 \mathrm{H}, \mathrm{NH}) \mathrm{ppm} .{ }^{13} \mathrm{C} \mathrm{NMR}(125 \mathrm{MHz}$, DMSO): $\delta 19.9,21.6,96.0,100.8,104.1,111.4,119.5,123.27,127.8,128.4,128.9,129.6,132.0,132.7,134.7$, 135.0, 161.8, 164.1, $167.7 \mathrm{ppm}$.

3-(3-(4-Chlorophenyl)-5-methoxy-1H-indol-2-yl)-4-hydroxy-6-methyl-2H-pyran-2-one (6c). Yellow powder; $\mathrm{mp} 272-275{ }^{\circ} \mathrm{C}$. IR v/cm ${ }^{-1}$ (KBr): 3401, 3094, 1649, $1484 \mathrm{~cm}^{-1} .{ }^{1} \mathrm{H}$ NMR (500 MHz, DMSO): $\delta 2.22\left(\mathrm{~s}, 3 \mathrm{H}, \mathrm{CH}_{3}\right)$, $3.69\left(\mathrm{~s}, 3 \mathrm{H}, \mathrm{OCH}_{3}\right), 6.05(\mathrm{~s}, 1 \mathrm{H}, \mathrm{ArH}), 6.60(\mathrm{~s}, 1 \mathrm{H}, \mathrm{ArH}), 6.74(\mathrm{~d}, J 9.5 \mathrm{~Hz}, 1 \mathrm{H}, \mathrm{ArH}), 7.27(\mathrm{~d}, J 8.5 \mathrm{~Hz}, 1 \mathrm{H}, \mathrm{ArH}), 7.42$ (d, J 8.5Hz, 2H, ArH), 7.55 (d, J 8.5Hz, 2H, ArH), 11.25 (s, 1H, NH) ppm. ${ }^{13} \mathrm{C}$ NMR (125 MHz, DMSO): $\delta$ 19.9, 55.9, 101.7, 112.4, 128.4, 128.9, 129.8, 130.4, 131.7, 131.8, 131.9, 132.0, 132.8, 135.1, 143.7, 145.7, 153.7, 161.7, 163.7 ppm. Anal. Calcd for $\mathrm{C}_{21} \mathrm{H}_{16} \mathrm{ClNO}_{4}$ (381.81): C, 66.06; H, 4.22; N, 3.67. Found: C, 66.12; H, 4.22; N, 3.70.

4-Hydroxy-6-methyl-3-(5-methyl-3-(4-nitrophenyl)-1H-indol-2-yl)-2H-pyran-2-one (6d). Yellow powder; mp 285-288 ${ }^{\circ} \mathrm{C}$. IR v/cm ${ }^{-1}(\mathrm{KBr}): 3413,3085,1645,1469 \mathrm{~cm}^{-1} .{ }^{1} \mathrm{H}$ NMR $(500 \mathrm{MHz}, \mathrm{DMSO}): \delta 2.00\left(\mathrm{~s}, 3 \mathrm{H}, \mathrm{CH}_{3}\right), 2.29$ $\left(\mathrm{s}, 3 \mathrm{H}, \mathrm{CH}_{3}\right), 5.41(\mathrm{~s}, 1 \mathrm{H}, \mathrm{ArH}), 6.88(\mathrm{~d}, J 8.0 \mathrm{~Hz}, 1 \mathrm{H}, \mathrm{ArH}), 6.94(\mathrm{~s}, 1 \mathrm{H}, \mathrm{ArH}), 7.20(\mathrm{~d}, J 8.0 \mathrm{~Hz}, 1 \mathrm{H}, \operatorname{ArH}), 7.95(\mathrm{~d}, J$ $9.0 \mathrm{~Hz}, 2 \mathrm{H}, \mathrm{ArH}), 8.13(\mathrm{~d}, J 9.0 \mathrm{~Hz}, 2 \mathrm{H}, \mathrm{ArH}), 8.49(\mathrm{~s}, 1 \mathrm{H}, \mathrm{OH}) 11.09(\mathrm{~s}, 1 \mathrm{H}, \mathrm{NH}) \mathrm{ppm} .{ }^{13} \mathrm{C} \mathrm{NMR}(125 \mathrm{MHz}, \mathrm{DMSO})$ : $\delta$ 20.0, 29.5, 102.4, 110.9, 123.7, 126.9, 137.9, 149.5, 150.5, 151.6, 153.6, 154.7, 155.2, 155.6, 157.1, 160.9, 161.8, 162.8, 178.2 ppm. Anal. Calcd for $\mathrm{C}_{21} \mathrm{H}_{16} \mathrm{~N}_{2} \mathrm{O}_{5}$ (376.37): C, 67.02; $\mathrm{H}, 4.29 ; \mathrm{N}, 7.44$. Found: $\mathrm{C}, 66.91 ; \mathrm{H}$, $4.27 ; \mathrm{N}, 7.38$. 
4-Hydroxy-3-(3-(4-methoxyphenyl)-5-methyl-1H-indol-2-yl)-6-methyl-2H-pyran-2-one (6e). Yellow powder; $\mathrm{mp} 133-136{ }^{\circ} \mathrm{C}$. IR v/cm ${ }^{-1}(\mathrm{KBr}): 3411,3001,1657,1448 \mathrm{~cm}^{-1} .{ }^{1} \mathrm{H}$ NMR (500 MHz, DMSO): $\delta 2.22\left(\mathrm{~s}, 3 \mathrm{H}, \mathrm{CH}_{3}\right)$, $2.32\left(\mathrm{~s}, 3 \mathrm{H}, \mathrm{CH}_{3}\right), 3.75\left(\mathrm{~s}, 3 \mathrm{H}, \mathrm{OCH}_{3}\right), 6.08(\mathrm{~s}, 1 \mathrm{H}, \mathrm{ArH}), 6.88(\mathrm{~d}, J 7.0 \mathrm{~Hz}, 1 \mathrm{H}, \mathrm{ArH}), 6.89(\mathrm{~s}, 1 \mathrm{H}, \mathrm{ArH}), 6.95(\mathrm{~d}, J$ $8.5 \mathrm{~Hz}, 2 \mathrm{H}, \mathrm{ArH}), 7.25(\mathrm{~d}, J 8.5 \mathrm{~Hz}, 1 \mathrm{H}, \mathrm{ArH}), 7.50(\mathrm{~d}, J 8.5 \mathrm{~Hz} 2 \mathrm{H}, \mathrm{ArH}), 11.16(\mathrm{~s}, 1 \mathrm{H}, \mathrm{NH}) \mathrm{ppm} .{ }^{13} \mathrm{C} \mathrm{NMR}(125$ $\mathrm{MHz}, \mathrm{DMSO}): \delta 19.9,21.7,55.5,96.4,100.6,102.3,111.0,114.3,119.0,122.9,126.4,127.5,128.0,129.9$, 134.8, 136.0, 158.9, 161.5, 164.3, 167.6 ppm. Anal. Calcd for $\mathrm{C}_{22} \mathrm{H}_{19} \mathrm{NO}_{4}$ (361.40): C, 73.12; $\mathrm{H}, 5.30 ; \mathrm{N}, 3.88$. Found: $\mathrm{C}, 73.20 ; \mathrm{H}, 5.34 ; \mathrm{N}, 3.86$.

4-Hydroxy-6-methyl-3-(5-chloro-3-(o-tolyl)-1H-indol-2-yl)-2H-pyran-2-one (6f). Yellow powder; mp 157-159 ${ }^{\circ} \mathrm{C}$. IR v $/ \mathrm{cm}^{-1}(\mathrm{KBr}): 3387,3007,1655,1445 \mathrm{~cm}^{-1} .{ }^{1} \mathrm{H}$ NMR $(500 \mathrm{MHz}, \mathrm{DMSO}): \delta 2.13\left(\mathrm{~s}, 3 \mathrm{H}, \mathrm{CH}_{3}\right), 2.21(\mathrm{~s}, 3 \mathrm{H}$, $\left.\mathrm{CH}_{3}\right), 5.93(\mathrm{~s}, 1 \mathrm{H}, \mathrm{ArH}), 6.63(\mathrm{~d}, J 8.5 \mathrm{~Hz}, 1 \mathrm{H}, \mathrm{ArH}), 6.89(\mathrm{~s}, 1 \mathrm{H}, \mathrm{ArH}), 6.94-7-05(\mathrm{~m}, 3 \mathrm{H}, \mathrm{ArH}), 7.10(\mathrm{~d}, J 8.0 \mathrm{~Hz}, 1 \mathrm{H}$, $\operatorname{ArH}), 7.26(\mathrm{t}, J 7.5 \mathrm{~Hz}, 1 \mathrm{H}, \mathrm{ArH}), 9.73(\mathrm{~s}, 1 \mathrm{H}, \mathrm{OH}), 10.84(\mathrm{~s}, 1 \mathrm{H}, \mathrm{NH}) \mathrm{ppm} .{ }^{13} \mathrm{C} \mathrm{NMR}(125 \mathrm{MHz}, \mathrm{DMSO}): \delta 21.1$, 22.7, 101.7, 109.4, 117.8, 120.6, 122.5, 124.7, 125.3, 126.8, 127.5, 128.7, 129.9, 131.7, 133.6, 135.7, 136.2, $136.8,161.6,165.3,167.2 \mathrm{ppm}$.

\section{References}

1. Borpatra, P. J.; Deka, B.; Rajbongshi, B. K.; Deb, M. L.; Baruah, P. K. Synthetic Commun. 2018, $48,2074$. https://doi.org/10.1080/00397911.2018.1482352

2. Badiger, J.; Manjulatha,K.; Girish, M.; Sharif A.; Purohit, M. G. Arkivoc 2009, xii, 217. https://doi.org/10.3998/ark.5550190.0010.c19

3. Wei, X.; Yang, J.; Ma, H. X.; Ding, C. F.; Yu, H. F.; Zhao, Y. L.; Liu, Y. P.; Khan, A.; Wang, Y. F.; Yang Z. F.; Huang, W. Y. Tetrahedron Lett. 2018, 59, 2066.

https://doi.org/10.1016/j.tetlet.2018.04.041

4. Huang, E.; Zhang, L.; Xiao, C.; Meng, G.; Zhang, B.; Hu, J.; Wan, D. C.; Meng, Q.; Jin Z.; Hu, C. Chinese Chem. Lett. 2019 Accepted.

https://doi.org/10.1016/i.cclet.2019.04.044

5. Suzdalev, K. F.; Popov, L. D.; Zubenko, A. A.; Drobin, Y. D.; Fetisov, L. N.; Bodryakov A. N.; Serbinovskaya, N. M. Russ. J. Bioorg. Chem. 2018, 44, 229.

https://doi.org/10.1134/S106816201801017X

6. Ali, R.; Saad, H. Molecules 2018, 23, 693.

https://doi.org/10.3390/molecules23030693

7. Zhao,Y. H.; Luo,Y.; Zhu, Y.; Wang, H.; Zhou, H.; Tan, H.; Zhou, Z.; Ma, Y. C.; Xie, W.; Tang, Z. Synlett. 2018, 29, 773.

https://doi.org/10.1055/s-0036-1591743

8. Kochanowska-Karamyan, A. J.; Hamann, M. T. Chem. Rev. 2010, 110, 4489. https://doi.org/10.1021/cr900211p

9. Ishikura, M.; Abe, T.; Choshi, T.; Hibino, S. Nat. Prod. Rep. 2015, 32, 1389. https://doi.org/10.1039/c5np00032g

10. Koch, K.; Campanella, C.; Baidoo, C. A.; Manzo, J. A.; Ameen, V. Z.; Kersey, K. E. Digest. Dis. Sci. 2004, 49, 1244.

https://doi.org/10.1023/B:DDAS.0000037819.31817.7c

11. Subbareddy, C. V.; Sumathi, S. New J. Chem. 2017, 41, 9388.

https://doi.org/10.1039/C7NJ00980A 
12. Kathrotiya, H. G.; Patel, M. P. Med. Chem. Res. 2012, 21, 3406.

https://doi.org/10.1007/s00044-011-9861-4

13. Nandakumar, A.; Thirumurugan, P.; Perumal, P. T.; Vembu, P.; Ponnuswamy, M. N.; Ramesh, P. Bioorg. Med. Chem. Lett. 2010, 20, 4252.

https://doi.org/10.1016/j.bmcl.2010.05.025

14. Kamath, P. R.; Sunil, D.; Ajees, A. A.; Pai, K. S.; Das, S. Bioorg. Chem. 2015, 63, 101.

https://doi.org/10.1016/j.bioorg.2015.10.001

15. Subbareddy,C. V.; Sundarrajan, S.; Mohanapriya, A.; Subashini, R.; Shanmugam, S. J. Mol. Liq. 2018, 251, 296.

https://doi.org/10.1016/i.molliq.2017.12.082

16. Saundane, A. R.; Vijaykumar, K.; Vaijinath, A. V. Bioorg. Med. Chem. Lett. 2013, 23, 1978.

http://dx.doi.org/10.1016/i.bmcl.2013.02.036

17. Reddy, B. S.; Reddy, M. R.; Madan, C. H.; Kumar, K. P.; Rao, M. S. Bioorg. Med. Chem. Lett. 2010, $20,7507$. http://dx.doi.org/10.1016/i.bmcl.2010.10.003

18. Fischer, E.; Jourdan, F. Ber. Dtsch. Chem. Ges. 1883, 16, 2241.

https://doi.org/10.1002/cber.188301602141

19. Hughes, D. L. Org. Prep. Proced. Intl. 1993, 25, 607.

https://doi.org/10.1080/00304949309356257

20. Campaigne, E.; Lake, R. D. J. Org. Chem. 1959, 24, 478.

https://doi.org/10.1021/jo01086a010

21. RajanBabu, T. V.; Chenard, B. L.; Petti, M. A. J. Org. Chem. 1986, 51, 1704.

https://doi.org/10.1021/jo00360a014

22. Bartoli, G.; Palmieri, G.; Bosco, M.; Dalpozzo, R. Tetrahedron Lett. 1989, 30, 2129.

https://doi.org/10.1016/S0040-4039(01)93730-X

23. Batcho, A. D.; Leimgruber, W. Org. Synth. 1985, 63, 214.

https://doi.org/10.1002/0471264180.0s063.28

24. Flanagan, J.C.; Dornan, L. M.; McLaughlin, M.; G. McCreanor, N.G.; Cook, M. J.; Muldoon, M. J. Green Chem. 2012, 14, 1281.

https://doi.org/10.1039/C2GC35062A

25. Dey, A.; Ali, M. A.; Jana, S.; Samanta, S.; Hajra,A. Tetrahedron Lett. 2017, 58, 313.

https://doi.org/10.1016/j.tetlet.2016.12.010

26. Muralirajan, K.; Cheng, C. H. Chem. Eur. J. 2013, 19, 6198.

https://doi.org/10.1002/chem.201300922

27. Shi, Z.; Glorius, F. Angew. Chem. Int. Edit. 2012, 51, 9220.

https://doi.org/10.1002/anie.201205079

28. Wang, C.; Sun, H.; Fang, Y.; Huang, Y. Angew. Chem. Int. Edit. 2013, 52, 5795.

https://doi.org/10.1002/anie.201301742

29. Zoller, J.; Fabry, D. C.; Ronge, M. A.; Rueping, M. Angew. Chem. Int. Edit. 2014, 53, 13264.

https://doi.org/10.1002/anie.201405478

30. Lin, W.; Zheng, Y. X.; Xun, Z.; Huang, Z. B.; Shi, D. Q. Acs. Comb. Sci. 2017, 19, 708.

https://doi.org/10.1021/acscombsci.7b00126

31. Jin, C.; Su, L.; Ma, D.; Cheng, M. New J. Chem. 2017;41, 14053.

https://doi.org/10.1039/C7NJ03144K

32. Volvoikar, P. S.; Tilve, S. G.; Tetrahedron Lett. 2018, 59, 1851. 
https://doi.org/10.1016/i.tetlet.2018.04.001

33. Darehkordi, A.; Rahmani, F.; Hashemi, V. Tetrahedron Lett. 2013, 54, 4689.

https://doi.org/10.1016/i.tetlet.2013.06.093

34. Cho, H.; Kim, H.; Lim, Y. J.; Lee, S. H. Arkivoc 2018, iii, 76.

https://doi.org/10.24820/ark.5550190.p010.280

35. Qiu, G.; Ding, Q.; Wu, J. Chem. Soc. Rev. 2013, 42, 5257.

https://doi.org/10.1039/C3CS35507A

36. Kumar, I.; Kumar, R.; Sharma, U. Synthesis 2018, 50, 2655.

https://doi.org/10.1055/s-0037-1609733; Art ID: ss-2018-m0041-r

37. Alizadeh-Bami, F.; Mehrabi, H.; Ranjbar-Karimi, R. J. Sulfur Chem. 2019, 40, 469. https://doi.org/10.1080/17415993.2019.1602127

38. Mehrabi,H.; Alizadeh-Bami, F.; Ranjbar-Karimi, R. Tetrahedron lett. 2018, 59, 1924.

https://doi.org/10.1016/i.tetlet.2018.03.093 